\title{
Synergistic Cytotoxic Effect of L-Asparaginase Combined with Decitabine as a Demethylating Agent in Pediatric T-ALL, with Specific Epigenetic Signature
}

\author{
Salvatore Serravalle, ${ }^{1}$ Salvatore N. Bertuccio, ${ }^{1}$ Annalisa Astolfi, ${ }^{1,2}$ \\ Fraia Melchionda, ${ }^{1}$ and Andrea Pession ${ }^{1}$ \\ ${ }^{1}$ Pediatric Hematology and Oncology, Department of Pediatrics, S.Orsola-Malpighi Hospital, University of Bologna, Bologna, Italy \\ "'Giorgio Prodi" Cancer Research Center, University of Bologna, Bologna, Italy
}

Correspondence should be addressed to Salvatore Serravalle; salvatoreserravalle@libero.it

Received 28 July 2016; Revised 27 October 2016; Accepted 10 November 2016

Academic Editor: Melchiorre Cervello

Copyright (c) 2016 Salvatore Serravalle et al. This is an open access article distributed under the Creative Commons Attribution License, which permits unrestricted use, distribution, and reproduction in any medium, provided the original work is properly cited.

T-Acute Lymphoblastic Leukemia (T-ALL) remains a subgroup of pediatric ALL, with a lower response to standard chemotherapy. Some recent studies established the fundamental role of epigenetic aberrations such as DNA hypermethylation, to influence patients' outcome and response to chemotherapy. Moreover, L-asparaginase is an important chemotherapeutic agent for treatment of ALL and resistance to this drug has been linked to ASNS expression, which can be silenced through methylation. Therefore, we tested whether the sensitivity of T-ALL cell lines towards L-asparaginase is correlated to the epigenetic status of ASNS gene and whether the sensitivity can be modified by concurrent demethylating treatment. Hence we treated different T-ALL cell lines with L-asparaginase and correlated different responses to the treatment with ASNS expression. Then we demonstrated that the ASNS expression was dependent on the methylation status of the promoter. Finally we showed that, despite the demethylating effect on the ASNS gene expression, the combined treatment with the demethylating agent Decitabine could synergistically improve the L-asparaginase sensitivity in those T-ALL cell lines characterized by hypermethylation of the ASNS gene. In conclusion, this preclinical study identified an unexpected synergistic activity of L-asparaginase and Decitabine in the subgroup of T-ALL with low ASNS expression due to hypermethylation of the ASNS promoter, while it did not restore sensitivity in the resistant cell lines characterized by higher ASNS expression.

\section{Introduction}

T-Acute Lymphoblastic Leukemia is an aggressive hematologic neoplasm which arises from the malignant transformation of T-cell progenitors and accounts for about $15 \%$ of pediatric ALL. Despite the encouraging results of modern chemotherapy ALL protocols, T-ALL remains, also in the pediatric setting, a subgroup with the worst prognosis [1, 2]. T-ALL can be divided into molecular genetic subgroups which are characterized by unique gene expression signatures and aberrant activation of specific T-ALL transcription factor oncogenes, including MEF2C, HOXA, TLX1, NKX2.1, TLX3, TAL1, LMO1, and LMO2, and of oncogenic signaling cascades, including interleukin 7 receptor (IL7R), Janus kinase (JAK), signal transducer and activator of transcription (STAT) [3], phosphatidylinositol 3kinase (PI3K)/Akt [4], and Ras/mitogen-extracellular signalregulated kinase (MEK)/extracellular signal-regulated kinase (ERK) [5]. On the other hand, various studies have shown an important role of epigenetic aberrations in the pathogenesis of pediatric acute leukemia, including T-ALL, and resistance to chemotherapy [6]. In particular, Borssen et al. recently showed that the amount of CpG island methylation could predict the outcome and response to standard treatment [7].

$\mathrm{L}$-asparaginase (L-Asp) is an important chemotherapeutic agent for the management of acute lymphoblastic leukemia. Resistance to L-Asp therapy may also be linked to high level of ASNS expression, which regulates the 
production of asparagine. Expression of cellular ASNS was found low in B-lineage ALL and T-lineage ALL, thus explaining the high sensitivity to L-Asp treatment $[8,9]$. In this regard, some studies described that the low expression of ASNS in certain subgroups of ALL is due to the hypermethylation of the ASNS promoter [10]. Nevertheless, the correlation of ASNS expression and sensitivity to L-Asp is actually discussed and highly controversial [11, 12].

Considering the strong correlation between ALL outcome and epigenetic aberrations such as DNA methylation, the aim of our study was to investigate a possible role of this epigenetic alteration in the sensitivity of T-ALL to LAsp and explore the efficacy of a combined treatment with demethylating agents that could modulate the sensitivity to L-Asp in this setting.

\section{Materials and Methods}

2.1. Cell Culture and Reagents. Human T-ALL cell lines, DND41, HPB-ALL, RPMI 8402, CCRF-CEM, and Jurkat, were grown in RPMI 1640 (Lonza), supplemented with $10 \%$ Fetal Bovine Serum (Invitrogen), $2 \mathrm{mM}$ L-glutamine (GIBCO), $100 \mathrm{U} / \mathrm{mL}$ penicillin, and $100 \mu \mathrm{g} / \mathrm{mL}$ streptomycin (GIBCO).

2.2. Treatment with L-Asparaginase and Decitabine. To test the effects of L-Asp, cell lines were cultured for $48 \mathrm{~h}$ in the presence of increasing drug concentrations from $10^{-5} \mathrm{U} / \mathrm{mL}$ to $10^{2} \mathrm{U} / \mathrm{mL}$.

To explore the efficacy of a combined treatment with demethylating agents, T-ALL cell lines were cultured and treated with $1 \mu \mathrm{M}$ of Decitabine for $24 \mathrm{~h}$. After $24 \mathrm{~h}$ cell lines were treated with increasing concentrations of L-Asp (from $10^{-5}$ to $10^{2}$ ) for $48 \mathrm{~h}$. Due to high instability, Decitabine was added every $24 \mathrm{~h}$.

L-asparaginase (Erwinase) was purchased from Jazz pharmaceutic; Decitabine instead was purchased from Sigma-Aldrich.

2.3. Cell Viability Assay. Cell viability was determined using the WST1 (4-[3-(4-lodophenyl)-2-(4-nitrophenyl)-2H-5-tetrazolio]-1,3-benzene disulfonate) cell proliferation kit (Roche Applied Science, Monza, Italy), according to manufacturer's instructions. Assays were performed in triplicate. Nonlinear regression curve fit and IC50 analysis were calculated using the GraphPad Prism software (San Diego, CA, USA). Statistical analysis was performed using Student's $t$-test at a significance level of $p<0.05$ (GraphPad Prism Software). For drug-combination experiments, a combination index (CI) was calculated using the Compusyn software. CI values $<1$ indicate synergism; CI $=1$ indicates an additive effect, whereas values $>1$ indicate antagonism.

2.4. RNA Extraction and Quantitative PCR ( $q R T-P C R)$. Total RNA was extracted by the RNeasy spin column method (Qiagen). $1 \mu \mathrm{g}$ total RNA was reversely transcribed to singlestranded cDNA using the Transcriptor first strand cDNA synthesis kit (Roche Diagnostics) with oligo-dT primers
$(2.5 \mu \mathrm{M})$. ASNS gene-specific primers were designed with Primer Express 3.0 Software (Applied Biosystems, Monza, Italy) and qRT-PCR was performed using FastStart Sybr Green (Roche Diagnostics) on the LightCycler 480 apparatus (Roche Diagnostics). DDCt method was used to quantify gene expression levels, relative to the two housekeeping genes GAPDH and ATP5B. Quantitative RT-PCR primer sequences were as follows: ASNS forward $\left(5^{\prime}\right.$ $\left.3^{\prime}\right)$ GACAGAAGGATTGGCTGCCT, ASNS reverse $\left(5^{\prime}-3^{\prime}\right)$ CATCCAGAGCCTGAATGCCT, GAPDH forward $\left(5^{\prime}-3^{\prime}\right)$ CCAATATGATTCCACCCATGGC, GAPDH reverse $\left(5^{\prime}-3^{\prime}\right)$ CTTGATTTTCGAGGGATCTCGC, ATP5B Forward $\left(5^{\prime}\right.$ $\left.3^{\prime}\right)$ GTCTTCACAGGTCATATGGGGA, ATP5B reverse $\left(5^{\prime}\right.$ $3^{\prime}$ )ATGGGTCCCACCATATAGAAGG. In order to prove the anticorrelation between ASNS expression and sensitivity to L-Asp, we calculated $r^{2}$ coefficient with GraphPad Prism software (San Diego, CA, USA). In the same way after $72 \mathrm{~h}$ of Decitabine $1 \mu \mathrm{M}$ treatment, we analyzed the expression of ASNS gene in the DND41 cell line.

2.5. Methylation Analysis. Genomic DNA was extracted using the QIAamp DNA kit (Qiagen). Methylation analysis was performed using the Epigentek Methylamp modification kit (Epigentek). After bisulfite modification, promoter region of the ASNS gene (position, CpG: 74 range $=$ chr7:97871872-97872607) was amplified with specific primers: ASNS forward primer $\left(5^{\prime}-3^{\prime}\right)$ TTAGGGAATTAGGATAGAAAGGTTT, ASNS reverse primer $\left(5^{\prime}-3^{\prime}\right)$ AAACAAACCAAATTCAAAAACCTCC $3^{\prime}$. PCR product was purified and labeled with BigDye Terminator 1.1 (Applied Biosystems) and sequenced on a ABI3730 instrument (Applied Biosystems).

\section{Results}

3.1. T-ALL Cell Lines Displayed Different Sensitivity to L-Asp Treatment. T-ALL cell lines were cultured with increasing concentrations of L-Asp, ranging from $0,0001 \mathrm{U} / \mathrm{mL}$ to $100 \mathrm{U} / \mathrm{mL}$ and cell viability was measured 48 hours after treatment.

The value of IC50 in T-ALL cell lines was between $10^{-2}$ and $10^{-4} \mathrm{U} / \mathrm{mL}$. In particular DND41, HPB-ALL, and RPMI 8402 were more sensitive than other T-ALL cell lines (Jurkat and CCRF-CEM) $(p=0.004)$ (Figure 1).

3.2. The Level of L-Asparaginase Sensitivity Correlated with ASNS Expression. The expression level of ASNS was evaluated by qRT-PCR. The analysis of results showed a variable expression of ASNS among T-ALL cell lines. In particular, cell lines displaying high sensitivity to L-Asp treatment had lower expression of the ASNS gene, whereas cell lines showing lower sensitivity to L-Asp treatment were characterized by higher expression of ASNS gene. Therefore in T-ALL cell lines the sensitivity to L-Asp treatment inversely correlated with the expression level of the ASNS gene ( $p=0.0003$ ), showing a mild positive correlation between the IC50 and the ASNS expression, with a $r^{2}$ of 0.6 (Figure 2). 


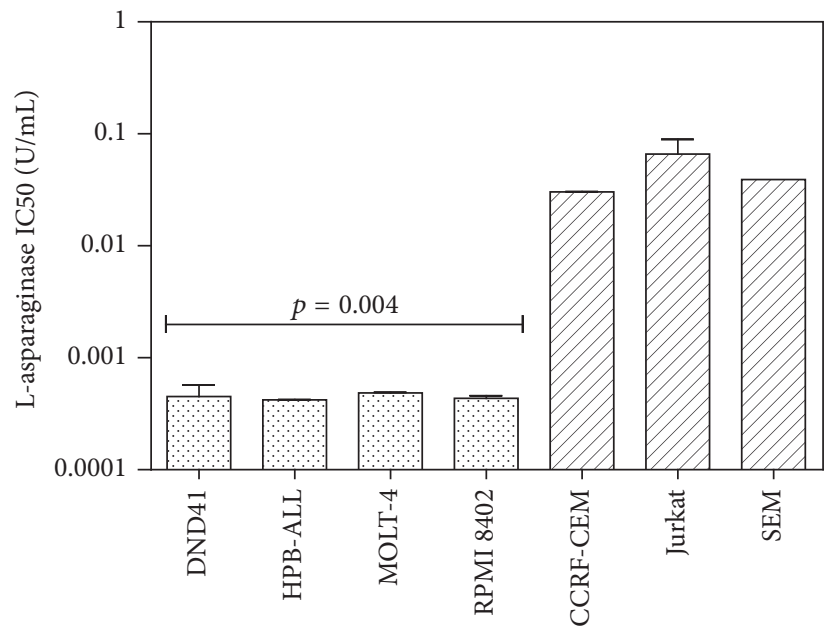

Figure 1: IC50 of L-asparaginase in T-ALL cell lines. DND41, HPB-ALL, and RPMI 8402 displayed higher sensitivity to L-Asp than other T-ALL cell lines $(p=0.004)$.

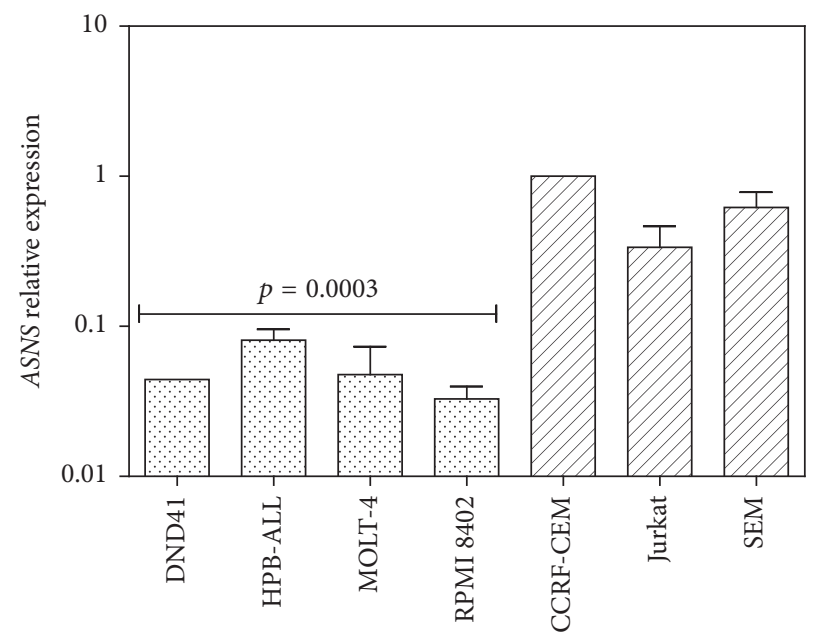

FIGURE 2: Expression analysis of ASNS gene in T-ALL cell lines. Cell lines that were more sensitive to L-Asp had lower expression of the ASNS gene than other T-ALL cell lines $(p=0.0003)$.

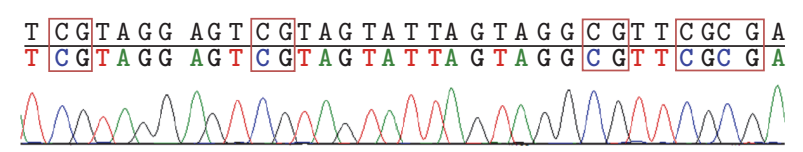

(a)
T GT A G G AG TT T TA G TA T TA G TA G GT GT T T GT G A T GTAG GAGTT TIAG TA T TA GTAGGT GT T T GT GA

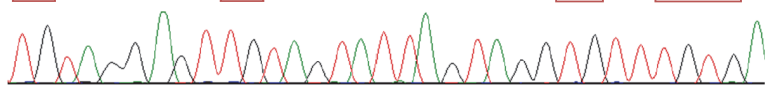

(b)

FIGURE 3: Example of cell lines with (a) hypermethylation of ASNS promoter and (b) cell lines without this epigenetic event.

3.3. Hypermethylation of the ASNS Promoter Correlated with ASNS Expression. To characterize the mechanism of the ASNS gene silencing in T-ALL cell lines, we analyzed the methylation status of the ASNS promoter. Sanger sequencing, performed on bisulfite-modified DNA, displayed a complete methylation of $\mathrm{CpG}$ islands in ASNS promoter in those cell lines which had a low expression of ASNS (HPB-ALL, DND41, and RPMI 8402) (Figure 3(a)). On the other hand,
Jurkat and CCRF-CEM cells did not display this epigenetic signature and showed a completely unmethylated promoter region (Figure 3(b)).

3.4. Decitabine Improved L-Asp Sensitivity in Cell Lines with Hypermethylation of ASNS CPG Islands. To better correlate the ASNS expression level with promoter methylation and check the effect of a demethylating agent to modulate L-Asp 

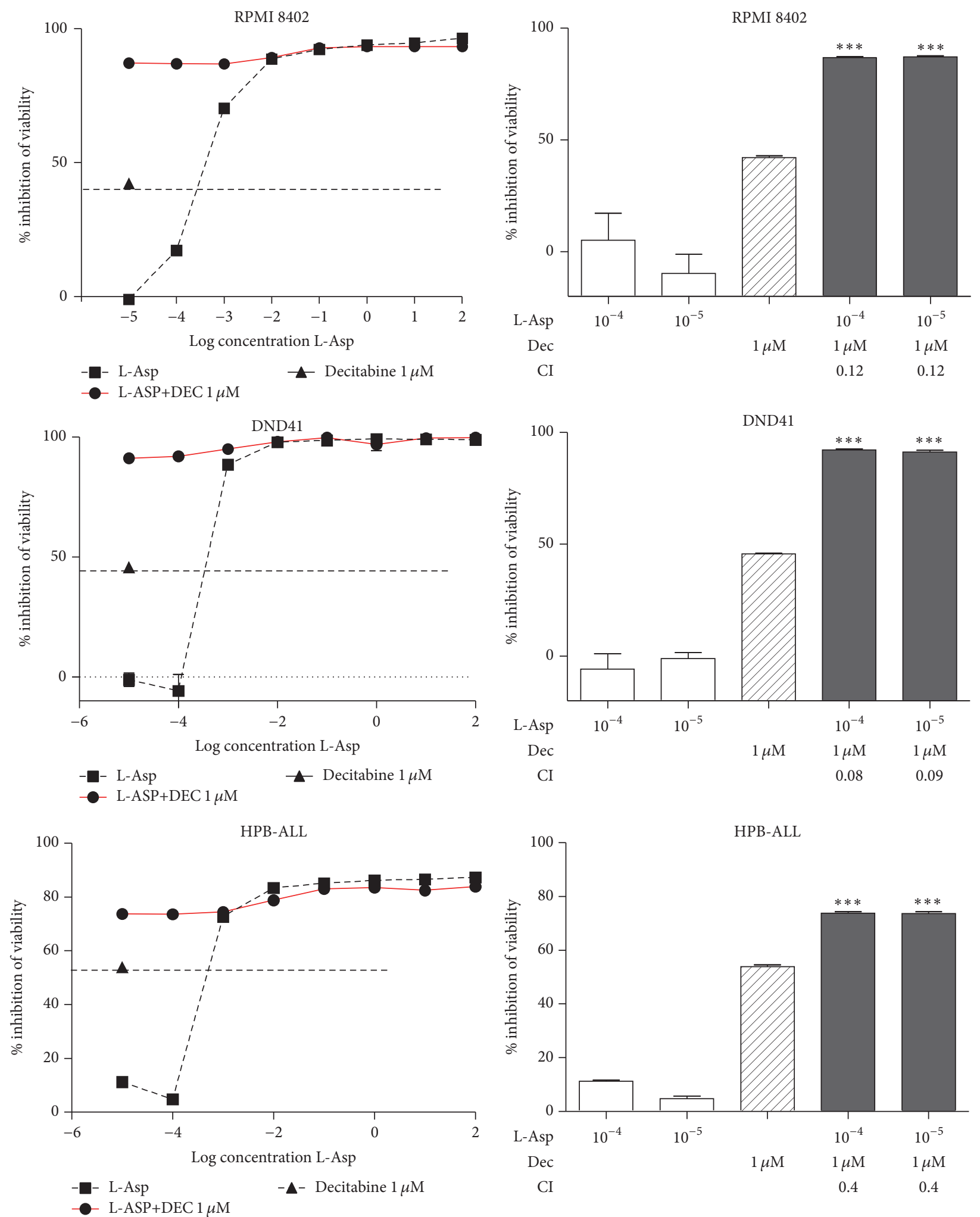

FIGURE 4: Synergic effect of Decitabine and L-Asp in RPMI 8402, DND41, and HPB-ALL. At $10^{-4}$ and $10^{-5} \mathrm{U} / \mathrm{mL}$ L-Asp alone did not affect cell viability, but addition of $1 \mu \mathrm{M}$ of Decitabine displayed a statistically significant synergic effect $\left({ }^{* * *} p\right.$ value $\left.<0.001\right)$. CI values $<1$ indicates synergism; CI $=1$ indicates additive effect, whereas values $>1$ indicates antagonism. 

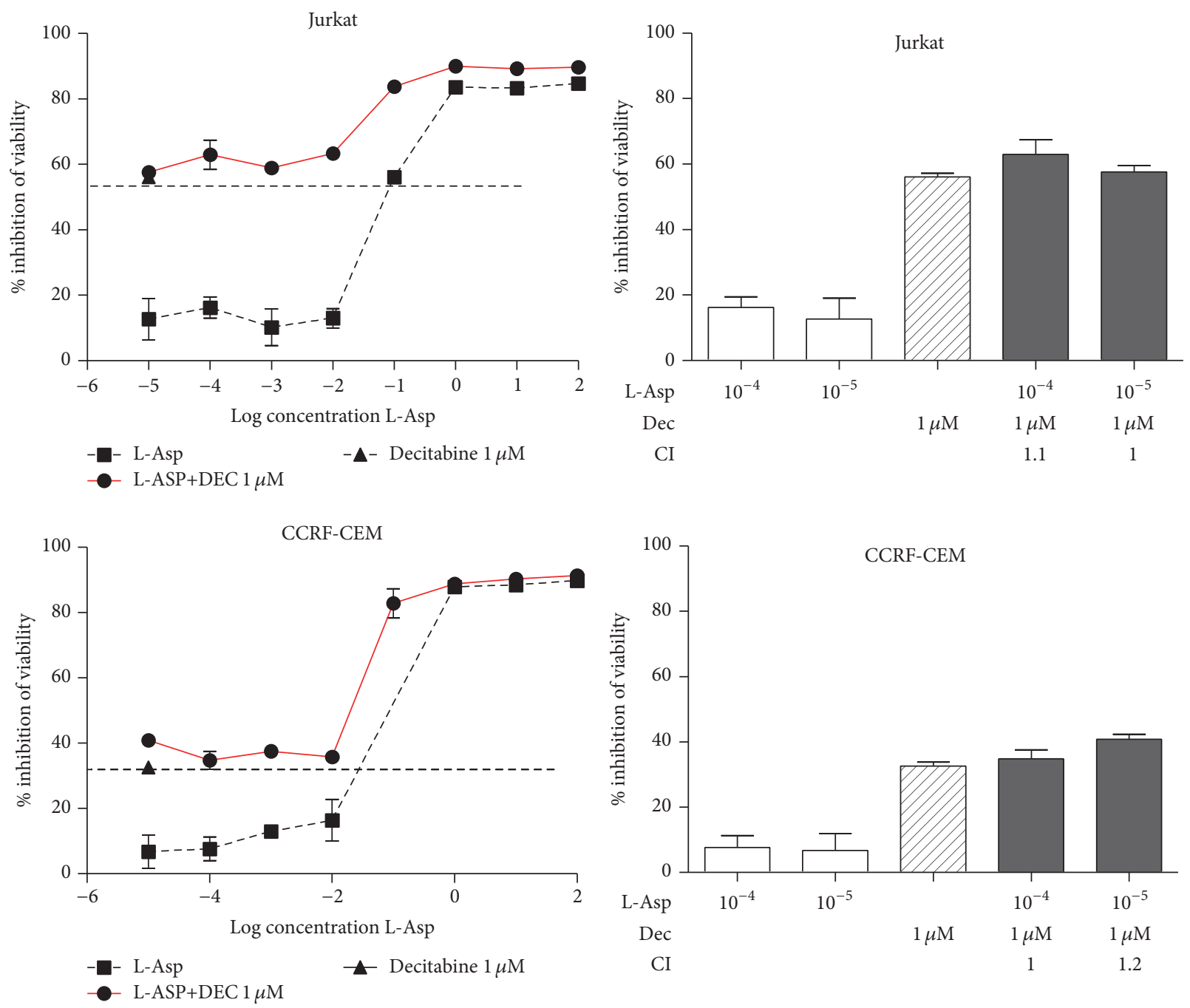

FIGURE 5: Jurkat and CCRF-CEM did not display synergism between Decitabine and L-asparaginase. CI values $<1$ indicate synergism; CI $=1$ indicates additive effect, whereas values $>1$ indicate antagonism.

sensitivity, we treated all T-ALL cell lines with Decitabine $1 \mu \mathrm{M}$, in combination with increasing concentrations of $\mathrm{L}$ Asp. Analysis of the results showed a reproducible toxicity in all T-ALL cell lines with about $50 \%$ of inhibition of cell viability. Unexpectedly, in cell lines displaying hypermethylation of ASNS promoter, Decitabine and L-Asp exhibited a strong synergistic effect with a value of combination index (CI) $<1$ (Figure 4). In particular, concentrations of $10^{-4} \mathrm{U} / \mathrm{mL}$ and $10^{-5} \mathrm{U} / \mathrm{mL}$ of L-Asp alone did not affect cell viability, but the percentage of citotoxicity increased dramatically in association with Decitabine. Other cell lines (Jurkat and CCRF-CEM) without methylation of ASNS promoter did not display this combined effect (CI $>1$ ) (Figure 5). To confirm the reactivation of ASNS expression after Decitabine treatment, we performed qPCR of ASNS gene in DND41 cell line. Following $72 \mathrm{~h}$ of treatment with $1 \mu \mathrm{M}$ of Decitabine, our data showed a significant increase in expression of the ASNS gene $(p<0.001)$ (Figure 6).

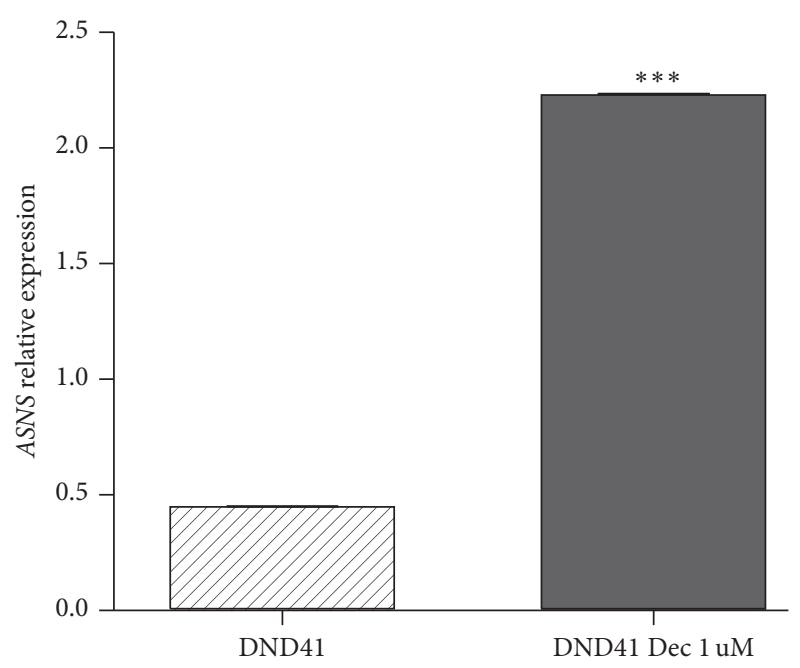

FIGURE 6: Expression of the ASNS gene in DND41 cell line after $72 \mathrm{~h}$ of treatment with $1 \mu \mathrm{M}$ of Decitabine. $\left({ }^{* * *} p\right.$ value $\left.<0.001\right)$. 


\section{Discussion and Conclusion}

T-ALL originates from T-cell precursors at different stages of their development and is characterized by distinct and well characterized molecular genetic subtypes. Children affected by this disease respond fairly well to high-dose chemotherapy regimens [1]. L-asparaginase is an important chemotherapeutic agent for the management of acute lymphoblastic leukemia. Resistance to L-Asp therapy may also be linked to high levels of ASNS cellular expression, which allows the production of asparagine. Expression of cellular ASNS is low in B-lineage ALL, explaining the higher sensitivity to L-Asp treatment. One mechanism implicated in gene silencing of ASNS is hypermethylation of the ASNS promoter. Moreover, in the last years some studies highlighted that epigenetic aberration may be determinant in inducing resistance to chemotherapy and poor outcome in T-ALL. Treatment with demethylating agents looks promising in an effort to provide new treatment options in T-cell malignant disorders [13]. In this context, our study attempted to investigate an epigenetic marker that could correlate with a high sensitivity to L-Asp and explored the interaction with demethylating agents such as Decitabine. Therefore we treated T-ALL cell lines with increasing concentrations of LAsp and found a subgroup of T-ALL cell lines displaying a high sensitivity to treatment. Then we confirmed that cell lines showing high sensitivity to L-Asp treatment had a very low expression of ASNS gene, which is in line with some literature data [10]. In our study, the mechanism of gene silencing in T-ALL cell lines displaying low expression of ASNS gene appeared to be linked to hypermethylation of the ASNS promoter as already shown [10]. Unexpectedly, we found a strong synergic effect of L-ASP and Decitabine, a demethylating agent, in cell lines with hypermethylation of ASNS at base line evaluation, DND41, HPB-ALL, and RPMI 8402.

This result seems to underline a different role of hypermethylation of ASNS promoter in the cellular response to L-Asp.

Therefore other studies are necessary to further explain the molecular mechanism underlying this result, which is probably linked to a larger set of methylated genes in $\mathrm{T}$ ALL subset. In particular, methylation status may not be restricted to ASNS gene and the reactivation of many genes such as oncosuppressors could have an important role in the synergic cytotoxic effect induced by Decitabine. The clinical translation of these data is not immediately predictable; however it remains an intriguing result because of the fact that cotreatment with a demethylating agent as Decitabine results in a synergistic effect. The role of demethylating agents in combination with standard chemotherapy should be further analyzed in preclinical and ex vivo models.

\section{Competing Interests}

The authors declare that there is no conflict of interests regarding the publication of this paper.

\section{References}

[1] K. Durinck, S. Goossens, S. Peirs et al., "Novel biological insights in T-cell acute lymphoblastic leukemia," Experimental Hematology, vol. 43, no. 8, pp. 625-639, 2015.

[2] P. Van Vlierberghe and A. Ferrando, "The molecular basis of T cell acute lymphoblastic leukemia," The Journal of Clinical Investigation, vol. 122, no. 10, pp. 3398-3406, 2012.

[3] D. Ribeiro, A. Melão, and J. T. Barata, "IL-7R-mediated signaling in T-cell acute lymphoblastic leukemia," Advances in Biological Regulation, vol. 53, no. 2, pp. 211-222, 2013.

[4] R. Fragoso and J. T. Barata, "Kinases, tails and more: regulation of PTEN function by phosphorylation," Methods, vol. 77-78, pp. 75-81, 2015.

[5] A. M. Martelli, G. Tabellini, F. Ricci et al., "PI3K/AKT/mTORC1 and MEK/ERK signaling in T-cell acute lymphoblastic leukemia: new options for targeted therapy," Advances in Biological Regulation, vol. 52, no. 1, pp. 214-227, 2012.

[6] R. Masetti, S. Serravalle, C. Biagi, and A. Pession, "The role of HDACs inhibitors in childhood and adolescence acute leukemias," Journal of Biomedicine and Biotechnology, vol. 2011, Article ID 148046, 9 pages, 2011.

[7] M. Borssen, Z. Haider, M. Landfors et al., "DNA methylation adds prognostic value to minimal residual disease status in pediatric T-cell acute lymphoblastic leukemia," Pediatric Blood \& Cancer, vol. 63, no. 7, pp. 1185-1192, 2016.

[8] S. Iwamoto, K. Mihara, J. R. Downing, C.-H. Pui, and D. Campana, "Mesenchymal cells regulate the response of acute lymphoblastic leukemia cells to asparaginase," The Journal of Clinical Investigation, vol. 117, no. 4, pp. 1049-1057, 2007.

[9] G. J. L. Kaspers, L. A. Smets, R. Pieters, C. H. Van Zantwijk, E. R. Van Wering, and A. J. P. Veerman, "Favorable prognosis of hyperdiploid common acute lymphoblastic leukemia may be explained by sensitivity to antimetabolites and other drugs: results of an in vitro study," Blood, vol. 85, no. 3, pp. 751-756, 1995.

[10] T. Akagi, D. Yin, N. Kawamata et al., "Methylation analysis of asparagine synthetase gene in acute lymphoblastic leukemia cells," Leukemia, vol. 20, no. 7, pp. 1303-1306, 2006.

[11] I. M. Appel, M. L. Den Boer, J. P. P. Meijerink, A. J. P. Veerman, N. C. M. Reniers, and R. Pieters, "Up-regulation of asparagine synthetase expression is not linked to the clinical response Lasparaginase in pediatric acute lymphoblastic leukemia," Blood, vol. 107, no. 11, pp. 4244-4249, 2006.

[12] N. Su, Y.-X. Pan, M. Zhou, R. C. Harvey, S. P. Hunger, and M. S. Kilberg, "Correlation between asparaginase sensitivity and asparagine synthetase protein content, but not mRNA, in acute lymphoblastic leukemia cell lines," Pediatric Blood and Cancer, vol. 50, no. 2, pp. 274-279, 2008.

[13] M. C. Kyrtsonis and D. Maltezas, "New evidence of gene inactivation by aberrant DNA-Methylation in T-cell leukemia, with treatment implications," Leukemia Research, vol. 50, pp. 29-30, 2016. 


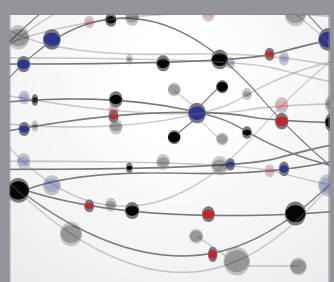

The Scientific World Journal
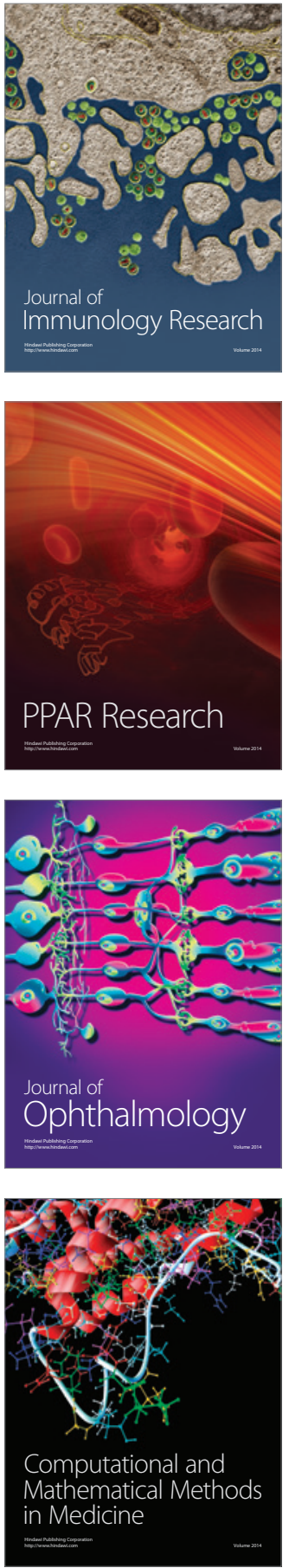

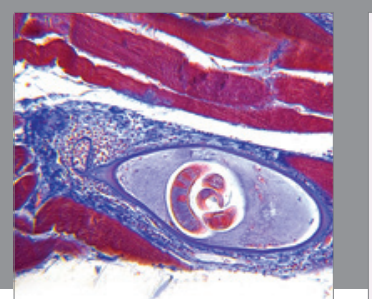

Gastroenterology Research and Practice

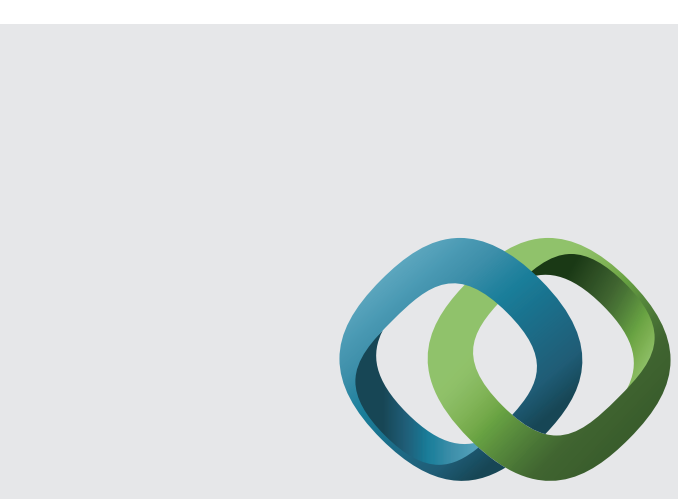

\section{Hindawi}

Submit your manuscripts at

http://www.hindawi.com
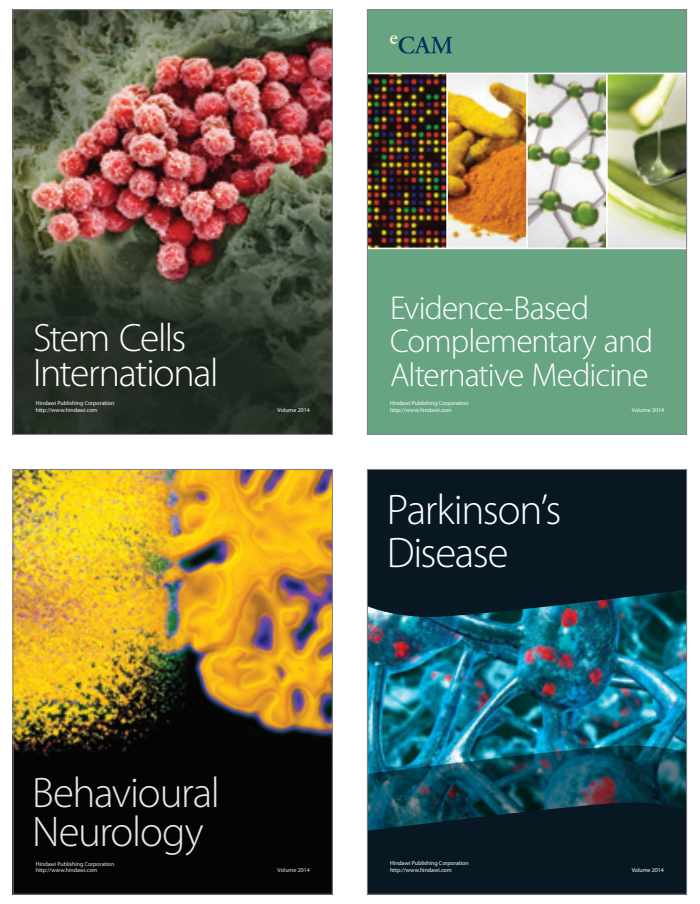
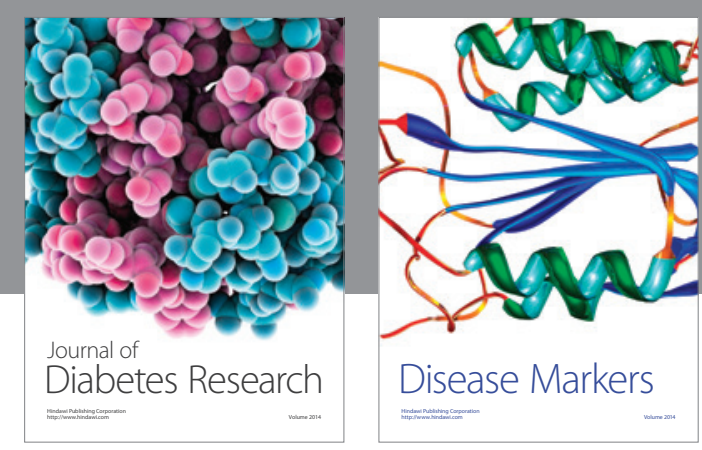

Disease Markers
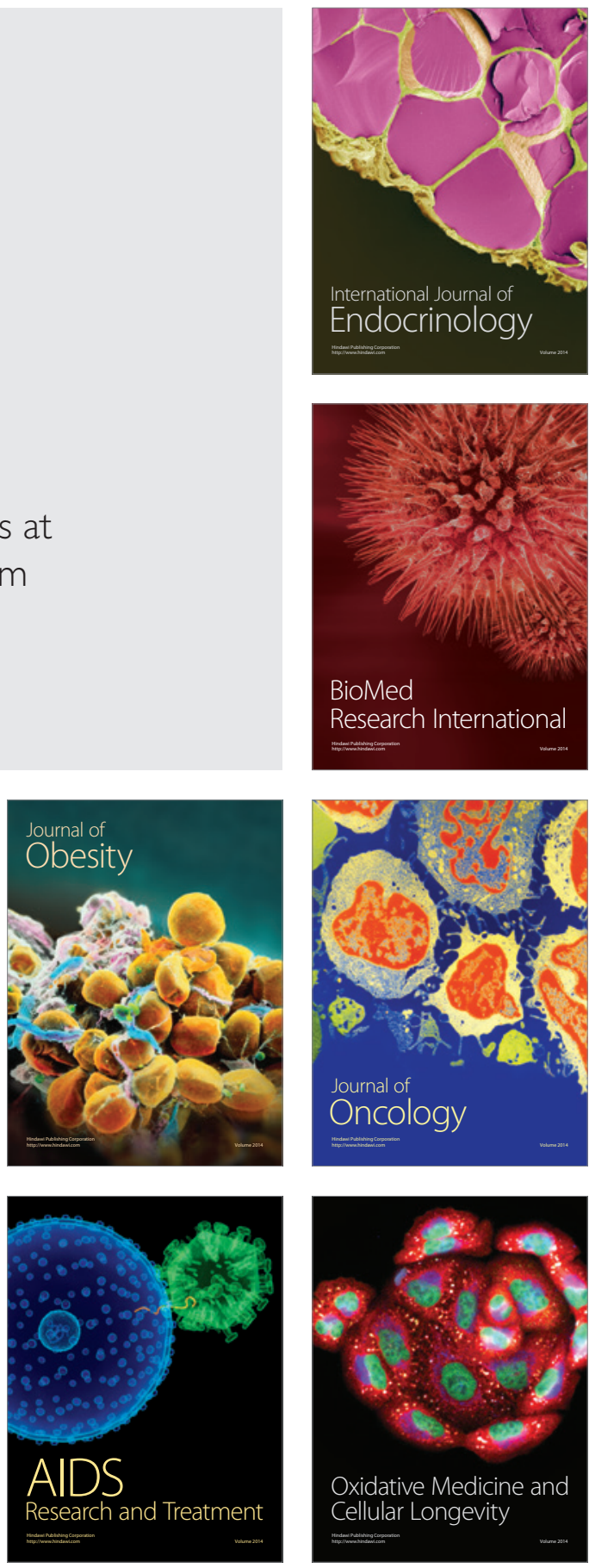